\title{
A Forward Scattering Dipole Model from a Functional Integral Approximation
}

\author{
ROALD FREDERICKX and PHILIP DUTRÉ, KU Leuven
}

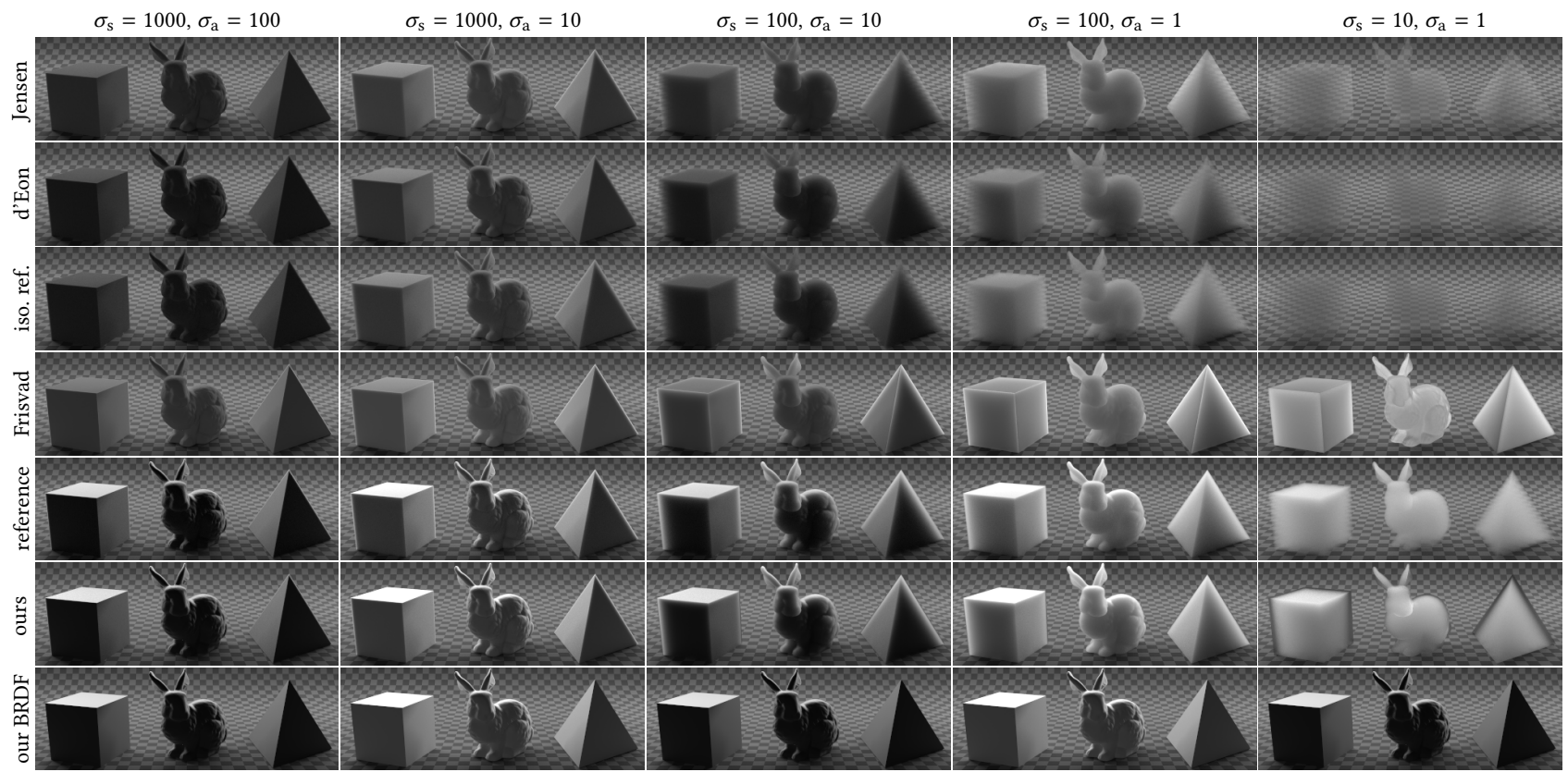

Fig. 1. Comparison of dipole models and ground truth images for an index matched medium with a forward peaked Gaussian phase function $(g=0.9)$ and varying medium densities. The first two rows show the dipole models of Jensen et al. [2001] and d'Eon [2014], respectively. The third row ('iso. ref.') gives a path traced ground truth image for an isotropic scattering function as determined from similarity theory (hence it is the ground truth image for the diffusion models above it). The fifth row ('Frisvad') shows the result of the directional dipole model of Frisvad et al. [2014], followed by the anisotropic reference solution ('reference'), with the last two rows showing the result of our forward scattering dipole model and effective BRDF, respectively. The scattering parameters are given above each of the columns and all objects are of unit size. Our subsurface scattering model accurately captures the ground truth behaviour over the entire range of parameters, and our BRDF provides a useful and computationally efficient approximation for the optically dense media on the left.

Rendering translucent materials with physically based Monte Carlo methods tends to be computationally expensive due to the long chains of volumetric scattering interactions. In the case of strongly forward scattering materials, the problem gets compounded since each scattering interaction becomes highly anisotropic and near-specular. Various well-known approaches try to avoid the resulting sampling problem through analytical approximations based on diffusion theory. Although these methods are computationally efficient, their assumption of diffusive, isotropic scattering can lead to considerable errors when rendering forward scattering materials, even in the

R. F. is a predoctoral fellow of the Fund for Scientific Research (FWO) of Flanders. Author's addresses: Roald Frederickx and Philip Dutré, Department of Computer Science, KU Leuven, Celestijnenlaan 200A, 3001 Leuven, Belgium. Email: \{roald.frederickx,philip.dutre\}@cs.kuleuven.be.

Permission to make digital or hard copies of all or part of this work for personal or classroom use is granted without fee provided that copies are not made or distributed for profit or commercial advantage and that copies bear this notice and the full citation on the first page. Copyrights for components of this work owned by others than ACM must be honored. Abstracting with credit is permitted. To copy otherwise, or republish, to post on servers or to redistribute to lists, requires prior specific permission and/or a fee. Request permissions from permissions@acm.org.

(c) 2017 ACM. 0730-0301/2017/7-ART109 $\$ 15.00$

DOI: http://dx.doi.org/10.1145/3072959.3073681 optically dense limit. In this paper, we present an analytical subsurface scattering model, derived with the explicit assumption of strong forward scattering. Our model is not based on diffusion theory, but follows from a connection that we identified between the functional integral formulation of radiative transport and the partition function of a worm-like chain in polymer physics. Our resulting model does not need a separate Monte Carlo solution for unscattered or single-scattered contributions, nor does it require ad-hoc regularization procedures. It has a single singularity by design, corresponding to the initial unscattered propagation, which can be accounted for by the extensive analytical importance sampling scheme that we provide. Our model captures the full behaviour of forward scattering media, ranging from unscattered straight-line propagation to the fully diffusive limit. Moreover, we derive a novel forward scattering BRDF as limiting case of our subsurface scattering model, which can be used in a level of detail hierarchy. We show how our model can be integrated in existing Monte Carlo rendering algorithms, and make comparisons to previous approaches. CCS Concepts: • Computing methodologies $\rightarrow$ Rendering; Ray tracing;

Additional Key Words and Phrases: subsurface scattering, forward scattering, participating media, radiative transport, BSSRDF, BRDF, rendering algorithms, global illumination. 


\section{ACM Reference format:}

Roald Frederickx and Philip Dutré. 2017. A Forward Scattering Dipole Model from a Functional Integral Approximation. ACM Trans. Graph. 36, 4, Article 109 (July 2017), 13 pages.

DOI: http://dx.doi.org/10.1145/3072959.3073681

\section{INTRODUCTION}

Translucent materials are ubiquitous in the natural world, ranging from minerals such as gemstones; weather effects such as fog, clouds and snow; organic substances such as fruit and many types of foods and drinks; and even our very own skin. The scattering of light within these substances gives rise to their characteristic appearance. Accurately rendering such effects within a physically based context is paramount for producing naturally looking images.

Standard Monte Carlo path tracing techniques simulate all individual scattering interactions, resulting in excessive computational costs for rendering densely scattering media. This problem gets aggravated when each scattering event becomes highly directional, as is the case for strong forward scattering. In such a setting, many advanced techniques that rely on path (re)connections start to degrade towards the performance of simple brute force path tracing. Indeed, each forward scattering event can be seen as a near-specular interaction, and long near-specular chains are a particularly challenging problem for many rendering algorithms [Jakob and Marschner 2012]. Apart from this angular singularity, there are also strong spatial constraints on path connections as highly anisotropic scattering usually goes hand in hand with high scattering densities (for a similar appearance, both can be thought of as inversely correlated through similarity theory [Wyman et al. 1989]). The result is a heavily suppressed throughput when connecting spatially separated points due to strong out-scattering.

Interestingly, many natural materials exhibit this problematic forward scattering behaviour [Gkioulekas et al. 2013; Narasimhan et al. 2006], including our own skin [Vo-Dinh 2003]. Hence, it is crucial to have efficient rendering methods for these types of common effects.

The seminal work of Jensen et al. [2001] introduced a fully analytical approximation of the subsurface light transport, which eliminates the need for a costly Monte Carlo sampling of the scattering interactions. This model arises from a diffusion approximation of the radiative transfer within the medium, and as such is strongly tied to the assumption of isotropic scattering.

In this paper, we introduce a model that explicitly starts from the assumption of strong forward scattering. Our approximation is based on the theory of functional integrals for radiative transfer [Tessendorf 2003], which we link to concepts from polymer physics in order to derive a practical rendering algorithm.

The contributions of this paper are the following:

- We derive an explicit translation between the functional integral formulation of forward scattering media and the partition function of a polymer with bending energy.

- We introduce a forward scattering dipole model, based on first principles, by exploiting this translation and adapting results from polymer physics.

- We show that our model is able to correctly reproduce the typical behaviour of forward scattering media, ranging from an initial straight-line, unscattered propagation over to the fully diffusive regime, which until now has not been possible with first-principle BSSRDF models.

- We present a forward scattering BRDF, derived from our subsurface scattering model, which can be used in a level of detail hierarchy.

Additionally, we provide extensive supplementary material that includes:

- A comprehensive set of analytical importance sampling schemes in order to use our model in practice.

- A step-by-step, self contained re-derivation of the functional integral formulation for forward scattering media.

This last derivation was first given by Tessendorf spread over a series of papers [1989; 1990; 1991; 2009], but we hope that an expanded, centralized re-derivation will help readers that are new to the subject.

\section{RELATED WORK}

\subsection{Radiative Transport}

Within computer graphics, the transport of light in scattering media is usually considered on a linearized mesoscopic scale as described by the Radiative Transfer Equation (RTE) [Modest 2013], thereby ignoring the wavelike nature of light and avoiding the full complexity of Maxwell's equations. The review of Cerezo et al. [2005] gives an overview of the problem and the initial techniques for rendering participating media. Two main approaches for solving the RTE have been adopted: Monte Carlo integration methods and photon density estimation methods, which have recently been unified into a single robust framework [Křivánek et al. 2014]. Nonetheless, strongly anisotropic scattering remains problematic, as each scattering event describes a near-specular interaction.

Although a perfect local importance sampling at each volumetric scattering event is possible, this does not lead to a globally optimal importance sampling [Křivánek and d'Eon 2014]. Georgiev et al. [2013] proposed to alleviate this problem through a joint importance sampling of up to two intermediate interactions in one step. However, generating the required sampling rules beyond a few intermediate vertices quickly becomes a formidable task, whereas such long paths do carry a significant contribution for strongly scattering media. Recent work of Weber et al. [2017] similarly tries to sample longer chains that connect to the light source in moderately dense, forward scattering media. Because their heuristic ignores correlations between successively generated vertices, this sampling strategy still has trouble with long paths in dense, highly forward scattering media. Alternatively, within a Markov Chain Monte Carlo context, Jakob and Marschner [2012] proposed a method whereby the near-specular volumetric paths get perturbed in a way that guarantees high throughput of the resulting path. However, for the long chains which are typical of a high albedo, strongly scattering medium, this technique can also become costly and susceptible to numerical problems.

A recent introduction within the graphics community has been the concept of zero variance random walks from the neutron transport literature [Krrivánek and d'Eon 2014; Meng et al. 2016]. This method uses an approximate solution of the radiance distribution 
to guide the path sampling process towards high-throughput paths As of yet, these methods have only been derived based on approximations for isotropically scattering media, and reduce to a standard unguided sampling in the limit of high anisotropy. Lifting these methods to anisotropic scattering media requires approximations of the radiance distribution tailored to that case. Note that the model that we describe in this work is, in fact, one such possibility for forward scattering media.

\subsection{Subsurface Scattering Models}

Approximate solution methods for the RTE based on diffusion theory are known from the neutron transport literature [Bell and Glasstone 1970]. Jensen et al. [2001] introduced these methods in computer graphics for rendering subsurface scattering in the form of a dipole based approximation to the Bidirectional Scattering Surface Reflection Distribution Function (BSSRDF) [Nicodemus et al. 1977]. This research has spurred many derivative works [d'Eon and Irving 2011; Donner and Jensen 2005; Li et al. 2005], which later started to take the directional dependence of incoming and outgoing rays into consideration [d'Eon 2014; Habel et al. 2013]. However, these methods are all based on isotropic scattering theory, and anisotropic scattering (e.g. forward scattering) is implemented through the use of similarity relations [Wyman et al. 1989; Zhao et al. 2014]. These relations are only valid towards the limit of diffusive transport and become inaccurate when this underlying assumption is violated.

In contrast to the purely isotropic methods, the directional dipole model of Frisvad et al. [2014] handles scattering anisotropy more explicitly. Through the use of the delta-Eddington approximation, the phase function is split into an unscattered delta distribution and a remaining linearly truncated phase function with reduced anisotropy. The unscattered incident light then acts as a ray source for the truncated phase function, which in turn gets handled by the diffusion approximation. Emergent light is assumed to be diffuse and hence the model is only sensitive to the incoming and not the outgoing direction.

Taking a different approach, Donner et al. [2009] generated a dense sampling of path traced Monte Carlo reference solutions across a large range of scattering parameters. The authors then proposed an empirical BSSRDF model, which was fitted to this data set and supports arbitrary scattering anisotropies. By contrast, our model is the result of a first-principles derivation and can trace its roots directly back to the radiative transfer equation, opening up a path towards deeper insights.

Lastly, some exact (semi-)analytical solution of Green's functions for the RTE are known. Paasschens [1997] found an expression in Fourier space for the time dependent Green's function of an isotropically scattering medium with isotropic point source, which was later used in a dipole BSSRDF setup by Martelli et al. [2007] For phase functions that only depend on the relative orientation of incoming and outgoing directions, Markel [2004] proposed a dimensional reduction by rotating the reference frame in the direction of the Fourier variable, resulting in a spherical harmonics series of the infinite-space Green's function for a directional point source. Liemert and Kienle [2013] additionally provide several exact solutions, including a solution for an anisotropic half-space with Fresnel boundary condition for a perpendicularly incident pencil beam in the form of a spherical harmonics expansion. The rotated reference frame idea of Markel was further used as a bridge from 3D transport theory to several known 1D transport solution methods by Machida [see Machida 2016, and references therein]. All of these methods provide exact solutions for the problems that they consider, but their numerical implementations quickly become overly costly for application in a rendering context.

\subsection{Functional Integrals for Radiative Transport}

Our forward scattering model derives from an approximation of the functional integral formulation of radiative transfer in participating media. Such functional integrals describe integrals of a functional over a function space and are well known in quantum field theory [Zee 2003]. Note that these functional integrals are also called path integrals, which should not be confused with the path integral framework by Veach [1998], as that still concerns integrals of ordinary functions over a space of path vertices.

The functional integral formalism for radiative transfer was introduced by Tessendorf [1987]. He provides an analytical approximation for the time indepdendent RTE that assumes a forward scattering phase function and a small deflection angle of the full path. This small-angle assumption is incompatible with a general subsurface scattering context, where incoming paths have to 'turn back around' over large angles to re-emerge out of the medium.

Tessendorf [1989; 1990] later expanded his work to the time dependent RTE in the form that we will use here. Exact analytical evaluations of the functional integral are still unfeasible and a Wentzel-Kramers-Brillouin (WKB) approximation was employed which expands the functional integral around the Most Probable Path (MPP) and considers only quadratic fluctuations around this MPP [Tessendorf 1991]. Within this same approximation, an effective multiply-scattered phase function was identified and used as a volumetric blurring scale in rendering [Tessendorf 2003; Tessendorf and Wasson 1994]. Further angular and spatial blurring results were derived from simplified MPPs and used for querying a precomputed, hierarchically blurred radiance cache during ray marching [Ashikhmin et al. 2004; Premože et al. 2003, 2004], as well as for real-time screen space scattering [Elek et al. 2013]. Lastly, a direct numerical integration of the full functional integral is also being investigated by representing paths as Frenet-Serret space curves [Kilgo and Tessendorf 2015; Tessendorf 2009].

\section{THE PROPAGATOR AS RTE SOLUTION KERNEL}

\subsection{Defining the Propagator}

Consider some volume of space $\mathcal{V} \subseteq \mathbb{R}^{3}$ filled with a participating medium. Light transport in this medium is governed by the time dependent radiative transfer equation (RTE), which reads [Modest 2013]

$$
\begin{aligned}
\left(\frac{\partial}{\partial s}+\hat{\omega} \cdot \vec{\nabla}_{\vec{x}}+\sigma_{\mathrm{t}}(\vec{x})\right) L(s, \vec{x}, \hat{\omega})= \\
Q(s, \vec{x}, \hat{\omega})+\sigma_{\mathrm{s}}(\vec{x}) \int P\left(\vec{x}, \hat{\omega}, \hat{\omega}^{\prime}\right) L\left(s, \vec{x}, \hat{\omega}^{\prime}\right) \mathrm{d}^{2} \hat{\omega}^{\prime} .
\end{aligned}
$$

Note that we work in units where the speed of light $c=1$ and have parametrized the transport in terms of path length $s=c t$ instead of 
time $t$. The RTE then describes how the radiance $L(s, \vec{x}, \hat{\omega})$ at 'time' $s$ at position $\vec{x} \in \mathcal{V}$ in direction $\hat{\omega}$ arises from a source $Q$ of emitted volumetric radiance density (dimensions of radiance per length). The properties of the medium are defined through the scattering phase function $P$ (normalized to unity) and the scattering and absorption coefficients ( $\sigma_{\mathrm{s}}$ and $\sigma_{\mathrm{a}}$, respectively) with $\sigma_{\mathrm{t}}=\sigma_{\mathrm{s}}+\sigma_{\mathrm{a}}$ being the transport or extinction coefficient.

Now, the radiative transfer equation is linear in the source term $Q$, and as such, the solution $L$ can be written as a convolution of the source $Q$ with a transport kernel or Green function $G$ :

$$
L(s, \vec{x}, \hat{\omega})=\int G\left(s-s^{\prime}, \vec{x}, \hat{\omega}, \vec{x}^{\prime}, \hat{\omega}^{\prime}\right) Q\left(s^{\prime}, \vec{x}^{\prime}, \hat{\omega}^{\prime}\right) \mathrm{d} s^{\prime} \mathrm{d} \vec{x}^{\prime} \mathrm{d}^{2} \hat{\omega}^{\prime} .
$$

The function $G\left(s-s^{\prime}, \vec{x}, \hat{\omega}, \vec{x}^{\prime}, \hat{\omega}^{\prime}\right)$ is also called the propagator and represents the differential fraction of radiance that reaches point $\vec{x}$ in the direction $\hat{\omega}$ after having started at point $\vec{x}^{\prime}$ in the $\hat{\omega}^{\prime}$ direction a time $s-s^{\prime}$ before. Concretely, it is the solution of the RTE with Dirac delta distribution source $Q(s, \vec{x}, \hat{\omega})=\delta\left(s-s^{\prime}\right) \delta^{3}\left(\vec{x}-\vec{x}^{\prime}\right) \delta^{2}\left(\hat{\omega}-\hat{\omega}^{\prime}\right)$ and has dimensions of (time $)^{-1} \times(\text { length })^{-3} \times(\text { solid angle })^{-1}$.

\subsection{Boundary Condition}

The RTE (1) is a first order (integro-)differential equation and as such its solution requires appropriate boundary conditions. The propagator $G$, being a particular solution of the RTE for a Dirac delta source, thus also requires its boundary conditions to be specified. These conditions couple the volumetric transport in $\mathcal{V}$ to surface scattering interactions on the boundaries $\partial \mathcal{V}$ of finite media (e.g. Fresnel reflection on an index mismatched boundary). For unbounded media, they determine the behaviour at infinity.

There are two approaches that we can take with respect to the boundary conditions of $G$. One option is to take the actual surface boundary conditions explicitly into account in $G$. This means that the propagator is fully aware of not only the shape and properties of the medium itself, but also the properties of its boundaries (e.g. how much light is reflected back into the medium, whether the reflections are specular, rough or diffuse, etc.). Keeping the volume properties fixed but changing the properties of the boundary then also causes the propagator $G$ to change.

A second option is to define $G$ with fully absorbing boundaries (i.e. outgoing light that crosses the boundary immediately gets absorbed and never reaches back into the medium). Any coupling to the actual boundary then has to be added externally and $G$ is kept independent of the true boundary conditions. This is the option that we will use throughout this work, as it facilitates the approximation of $G$ and we found this to yield better results for strongly forward scattering media with refractive boundaries near optically thin regions.

Specifically, the boundary condition on $G$ reads

$$
G\left(s-s^{\prime}, \vec{x}, \hat{\omega}, \vec{x}^{\prime}, \hat{\omega}^{\prime}\right)=0 \quad \text { for } \quad \vec{x} \in \partial \mathcal{V} \text { and } \hat{\omega} \cdot \hat{n}(\vec{x})<0,
$$

where $\hat{n}(\vec{x})$ denotes the outward-facing normal of the boundary at the point $\vec{x} \in \partial \mathcal{V}$ and the condition $\hat{\omega} \cdot \hat{n}(\vec{x})<0$ selects incoming directions at the boundary .

\subsection{Rendering Equation}

Because we defined the propagator to be unaware of the actual boundary conditions, we have to impose them explicitly during rendering. Coupling $G$ to the true boundary happens through a suitable choice of the source $Q$, for which we provide a full derivation in the supplementary material. The result is the following rendering equation for the incident radiance $L_{\mathrm{i}}$ :

$$
\begin{aligned}
& L_{\mathrm{i}}(s, \vec{x}, \hat{\omega})=\int \mathrm{d} s^{\prime} \mathrm{d} A\left(\vec{x}^{\prime}\right) \mathrm{d}^{2} \hat{\omega}^{\prime} G\left(s-s^{\prime}, \vec{x},-\hat{\omega}, \vec{x}^{\prime}, \hat{\omega}^{\prime}\right)\left|\hat{\omega}^{\prime} \cdot \hat{n}\left(\vec{x}^{\prime}\right)\right| \\
& \times\left[L_{\mathrm{e}}\left(s^{\prime}, \vec{x}^{\prime}, \hat{\omega}^{\prime}\right)+\int \mathrm{d}^{2} \hat{\omega}^{\prime \prime} L_{\mathrm{i}}\left(s^{\prime}, \vec{x}^{\prime}, \hat{\omega}^{\prime \prime}\right) f_{\mathrm{s}}\left(\vec{x}^{\prime}, \hat{\omega}^{\prime \prime} \leftrightarrow \hat{\omega}^{\prime}\right)\left|\hat{\omega}^{\prime \prime} \cdot \hat{n}\left(\vec{x}^{\prime}\right)\right|\right]
\end{aligned}
$$

where $f_{\mathrm{s}}$ is the BSDF of the boundary and we use the common convention that $L_{\mathrm{i}}(s, \vec{x}, \hat{\omega})$ defines the radiance that is incident on a surface from direction $\hat{\omega}$ (i.e. $\hat{\omega}$ pointing from receiver to source).

In our volumetric setting, $L_{\mathrm{i}}$ represents the radiance that is incident upon the inside surface of the medium due to volumetric scattering from within the medium. In other words, an extra BSDF evaluation will be needed to transform $L_{\mathrm{i}}$ on the inside to $L_{\mathrm{O}}$ on the outside of the object when rendering. How Eq. (4) is used in a rendering context will be explained in more detail in Sec. 7 .

Lastly, we refer the interested reader to the supplementary material for a deeper discussion of the propagator and the relationship between its corresponding linear operator formalism and the operator formalism of Jakob [2013], as well as a formal construction of the BSSRDF in terms of $G$.

\section{THE FORWARD SCATTERING PROPAGATOR}

Tessendorf [1989] showed that the propagator $G$ for the time dependent RTE can in general be expressed as a functional integral in Fourier transformed space. For strongly forward scattering media, an approximate analytical Fourier inversion is possible to obtain a functional integral for a forward scattering propagator [Tessendorf 1991, 2003]. We skip straight to the main results and refer to the supplementary material for an expanded re-derivation and a discussion of the functional integral regularization.

\subsection{Functional Integral Approximation}

The derivation of the functional integral result assumes a simple forward peaked Gaussian phase function of the form

$$
P_{\text {gauss }}\left(\hat{\omega}, \hat{\omega}^{\prime}\right)=N \exp \left(-\frac{\left(\hat{\omega}-\hat{\omega}^{\prime}\right)^{2}}{2 \mu}\right)=N \exp \left(-\frac{1-\hat{\omega} \cdot \hat{\omega}^{\prime}}{\mu}\right),
$$

where $\mu$ is a parameter that determines the width of the phase function and $N$ is a normalization constant such that $\int \mathrm{d} \hat{\omega}^{\prime} P\left(\hat{\omega}^{\prime}, \hat{\omega}\right)=$ $\int \mathrm{d} \hat{\omega}^{\prime} P\left(\hat{\omega}, \hat{\omega}^{\prime}\right)=1$ for all $\hat{\omega}$. For a heavily forward peaked phase function $(\mu \ll 1)$, the mean cosine $g$ of the deflection angle is given by $g \approx 1-\mu$, and hence the reduced scattering coefficient $\sigma_{\mathrm{s}}^{\prime} \equiv(1-g) \sigma_{\mathrm{s}}$ is given as $\sigma_{\mathrm{s}}^{\prime}=\mu \sigma_{\mathrm{s}}$ in this limit (a general relation of $\mu$ in terms of $g$ is given in the supplementary material).

For the forward peaked Gaussian phase function of Eq. (5), a corresponding approximate solution for the propagator $G$ can be given in terms of the functional integral

$$
\begin{aligned}
& G\left(s, \vec{x}, \hat{\omega}, \vec{x}^{\prime}, \hat{\omega}^{\prime}\right)=N_{G} e^{-\sigma_{\mathrm{a}} s} \int[D \hat{\beta}] \delta^{2}\left[\hat{\beta}(0)-\hat{\omega}^{\prime}\right] \delta^{2}[\hat{\beta}(s)-\hat{\omega}] \\
& \quad \times \delta^{3}\left[\vec{x}-\vec{x}^{\prime}-\int_{0}^{s} \mathrm{~d} s^{\prime} \hat{\beta}\left(s^{\prime}\right)\right] \exp \left[-\frac{1}{2 \sigma_{\mathrm{s}}^{\prime}} \int_{0}^{s} \mathrm{~d} s^{\prime}\left|\frac{\mathrm{d} \hat{\beta}\left(s^{\prime}\right)}{\mathrm{d} s^{\prime}}\right|^{2}\right]
\end{aligned}
$$


where $N_{G}$ is a normalization factor and $\int[D \hat{\beta}]$ denotes an integration over all functions $\hat{\beta}\left(s^{\prime}\right)$ representing all possible curved paths that a ray of light can follow through a scattering medium. The parametrization of the path is such that $\hat{\beta}\left(s^{\prime}\right)$ represents the unit-length tangent vector of the path at 'time' $s$ ', with time being parametrized in path length. The delta distributions in Eq. (6) then enforce the initial and final direction constraints and the end-to-end displacement constraint, respectively.

\subsection{Length Constraint Problem}

Apart from the delta distributions in Eq. (6), the only dependence on the shape of the path comes from the factor

$$
\exp \left[-\frac{1}{2 \sigma_{\mathrm{s}}^{\prime}} \int_{0}^{s} \mathrm{~d} s^{\prime}\left|\frac{\mathrm{d} \hat{\beta}\left(s^{\prime}\right)}{\mathrm{d} s^{\prime}}\right|^{2}\right],
$$

which determines the relative weight of each path. Working in a mechanical analogy, the argument of the exponential can be thought of as a quadratic bending energy that tries to keep deviations in the direction of the path small. Indeed, paths that show sharp deviations will have to scatter over large angles, and hence be heavily suppressed by a forward scattering phase function.

When a quadratic bending energy is exponentiated as in Eq. (7), the result is a simple Gaussian functional, for which functional integrals can be calculated exactly [Zee 2003]. However, the problem here lies in the requirement that $\hat{\beta}\left(s^{\prime}\right)$ remains a unit vector.

Initial work simply ignored the unit-length constraint on $\hat{\beta}\left(s^{\prime}\right)$, which was only satisfactory in some specific regimes [Tessendorf 1991]. Tessendorf's later work [2003] recognizes an exact solution of the path integral with the $\left|\hat{\beta}\left(s^{\prime}\right)\right|=1$ constraint in terms of an infinite sum of spherical harmonics, but this evaluation ignores the constraint on the displacement of the paths $\left(\int_{0}^{s} \hat{\beta}\left(s^{\prime}\right) \mathrm{d} s^{\prime}=\vec{x}-\vec{x}^{\prime}\right)$ and effectively behaves as a modified multiple scattering phase function without dependence on $\vec{x}-\vec{x}^{\prime}$. We employ a different approximation that keeps the explicit directional and displacement constraints intact, by exploiting a connection to polymer physics.

\subsection{Connection with polymer physics}

We make the interpretation of the weight in Eq. (7) as a bending energy exact by introducing a direct link with the partition function $Z$ in statistical mechanics (in particular statistical field theory). Such a partition function can roughly be interpreted as counting the number of states that are thermally accessible to a system [Kardar 2007; Zee 2003]. For a classical system, the partition function has the general form

$$
Z=\int \exp \left(-\frac{1}{k_{\mathrm{B}} T} H(\text { conf })\right) \mathrm{d}(\text { conf }),
$$

where $T$ is the temperature, $k_{\mathrm{B}}$ is the Boltzmann constant and $H$ (conf) is the Hamiltonian (i.e. energy) of the configuration and the integral is over the possible configurations of the system (e.g. the set of positions and momenta).

In our case, the configurations of the system are the possible paths $\hat{\beta}\left(s^{\prime}\right)$ that obey the given initial and final directions and the specified overall displacement of $\vec{x}-\vec{x}^{\prime}$. The (bending) energy of one such configuration is then proportional to $\int_{0}^{s} \mathrm{~d} s^{\prime}\left|\mathrm{d} \hat{\beta}\left(s^{\prime}\right) / \mathrm{d} s^{\prime}\right|^{2}$.
These kinds of functional integrals also appear as models of stiff polymers in the polymer physics literature [Freed 1972] and in fact we can write a direct translation between the scattering parameters of our path integral and the persistence length $l_{\mathrm{p}}$ of the wormlike chain model as $l_{\mathrm{p}} \leftrightarrow \frac{1}{\sigma_{\mathrm{s}}^{\prime}}$.

Mostly, the polymer physics literature is not directly concerned with the initial and final directions of the polymer (but only in the distance between the end points, for instance), and these directional quantities are often integrated out in the partition functions under consideration. However, some authors do take the end directions into account. Spakowitz and Wang [2005], for instance, have managed to find an analytical solution in Fourier-Laplace space for the exact path integral that we are considering. Although the result is impressive, we find the evaluation of the model too costly to incorporate into a practical rendering system.

\section{PRACTICAL APPROXIMATION OF INFINITE VOLUME FORWARD SCATTERING PROPAGATOR}

In the supplementary material, we use the link with polymer physics and a result of Winkler et al. [1994] as a starting point to obtain an approximate evaluation of the functional integral in Eq. (6) through a modified $\left|\vec{\beta}\left(s^{\prime}\right)\right|=1$ length constraint. The final form of the infinite volume forward scattering propagator $G_{\mathrm{f}}$ reads:

$$
\begin{aligned}
& G_{\mathrm{f}}\left(s, \vec{x}, \hat{\omega}, \vec{x}^{\prime}, \hat{\omega}^{\prime}\right)= \\
& N \exp \left(-C-D \hat{\omega} \cdot \hat{\omega}^{\prime}+E \vec{r} \cdot\left(\hat{\omega}+\hat{\omega}^{\prime}\right)-F|\vec{r}|^{2}-\sigma_{\mathrm{a}} s\right), \\
& \text { where } p=\frac{\sigma_{\mathrm{s}}^{\prime}}{2}=\frac{(1-g) \sigma_{\mathrm{s}}}{2}, \\
& \vec{r}=p\left(\vec{x}-\vec{x}^{\prime}\right), \\
& A=(p s-\tanh (p s))^{-1}, \\
& B=\frac{\tanh (p s)}{2}, \\
& C=\frac{9}{2 p s}, \\
& D=3 A B^{2}-\frac{3}{2 \sinh (2 p s)} \\
& E=3 A B, \\
& F=\frac{3}{2} A, \\
& N=p^{3} \frac{\sqrt{F}\left(E^{2}-2 D F\right)}{4 \pi^{5 / 2}\left(e^{E^{2} / F-D}-e^{D}\right)} \exp (C) .
\end{aligned}
$$

The factors ${ }^{1} A, B, C, D, E, F$, are all positive, dimensionless quantities that only depend on $p s$, with $p$ acting as a scale factor with dimension of inverse length.

For fixed path lengths $s$, the propagator $G_{f}$ of Eq. (9) behaves as a simple Gaussian distribution on position for fixed directions (similar to the usage of the time dependent diffusion equation in quantized diffusion [d'Eon and Irving 2011]) and as an exponential

\footnotetext{
${ }^{1}$ Purely mathematically, the $-C$ term in the exponential weight is superfluous as it gets cancelled by the $\exp (C)$ factor in the normalization $N$, but its purpose is to improve the numerical stability by cancelling an exponential divergence in $N$ as $s \rightarrow 0$.
} 
distribution on the outgoing directional cosine $\hat{\omega} \cdot\left(E \vec{r}-D \hat{\omega}^{\prime}\right)$ for fixed displacement $\vec{r}$ and incoming direction $\hat{\omega}^{\prime}$.

\subsection{Asymptotic Behaviour}

It is instructive to examine the asymptotic behaviour of Eq. (9). In this section, we state results for the short and diffusive limit. The supplementary material provides derivations and additional results.

For path lengths much smaller than the reduced scattering length (i.e. $p s \ll 1$ ), we expect the propagator to approach an unscattered delta distribution. At first sight, the sign of the $-D \hat{\omega} \cdot \hat{\omega}^{\prime}$ term in the exponential weight of Eq. (9) might seem somewhat alarming, as it apparently forces $\hat{\omega}$ and $\hat{\omega}^{\prime}$ towards opposite directions. However, this originates from an interplay with a weight that forces the displacement $\vec{R} \equiv \vec{x}-\vec{x}^{\prime}$ to align with $\hat{\omega}$ and $\hat{\omega}^{\prime}$. Indeed, for $p s \ll 1$, Eq. (9) expands to

$$
\frac{p^{3} 81 \sqrt{2}}{32 \pi^{5 / 2}(p s)^{11 / 2}} \exp \left(-\frac{9}{2 p s}\left(\frac{\vec{R}}{s}-\frac{\hat{\omega}+\hat{\omega}^{\prime}}{2}\right)^{2}-\frac{3}{8 p s}\left(\hat{\omega}-\hat{\omega}^{\prime}\right)^{2}\right) \text {. }
$$

The result is a Gaussian weight forcing $\vec{R} / s$ to point along the average direction $\left(\hat{\omega}+\hat{\omega}^{\prime}\right) / 2$, and a slightly smaller weight forcing both directions to align with each other. In the limit of $p s \rightarrow 0$, the only contribution comes from $\hat{\omega}=\hat{\omega}^{\prime}$ and $\vec{R}=s \hat{\omega}=s \hat{\omega}^{\prime}$, which is exactly the behaviour of an unscattered propagator.

With regards to the long time behaviour $p s \gg 1$, we can show that the fluence $\phi$ of an isotropic point source at position $\vec{x}^{\prime}$ approaches

$$
\phi(\vec{x}, s)=\left(\frac{3 p}{2 \pi s}\right)^{3 / 2} \exp \left(-\frac{3 p}{2 s}\left|\vec{x}-\vec{x}^{\prime}\right|^{2}-\sigma_{\mathrm{a}} s\right)
$$

under the assumptions (1) ps $\gg 1$ and (2) $s \gg\left|\vec{x}-\vec{x}^{\prime}\right|$. In other words, paths should be sufficiently long to ensure they (1) scatter multiple times until they become diffused, and (2) can reach the query point and, moreover, are long past the initial ballistic peak. Diffusion theory is expected to be valid in this regime, and Eq. (11) indeed corresponds to the classical result for the time dependent diffusion equation [Chandrasekhar 1943; Patterson et al. 1989].

Overall, our propagator can capture the correct qualitative behaviour over the entire spectrum ranging from unscattered straightline propagation to the fully diffusive limit.

\subsection{A Note on Similarity Theory}

The only dependence of Eq. (9) on the medium scattering properties ( $\sigma_{\mathrm{s}}$ and the phase function) comes in the form of the scaling factor $p=\sigma_{\mathrm{s}}^{\prime} / 2$. This dependence on the reduced scattering density $\sigma_{\mathrm{s}}^{\prime}$ is reminiscent of how anisotropy is handled within the diffusion approximation. Indeed, in the same manner that diffusion theory can be thought of as an expansion up to the lowest nontrivial order around an isotropically scattering phase function $(g=0)$, our approach can be viewed as an analogous expansion around a maximally forward scattering phase function $(g \rightarrow 1)$. In these lowest order approaches, deviations from the base assumption $(g=0$ or $g \rightarrow 1)$ are both encoded through a single, scalar value: the reduced scattering density $\sigma_{\mathrm{s}}^{\prime}=(1-g) \sigma_{\mathrm{s}}$, which is consistent with the predictions of similarity theory [Wyman et al. 1989].

As a remark, this observation opens up a natural avenue for future work: moderately forward phase functions that are too anisotropic to be accurately handled by the diffusion equation, but not sufficiently forward scattering to be handled purely with our model, might benefit from an interpolation between an isotropic BSSRDF and our forward scattering subsurface model. A related preliminary result is provided in the supplementary material for the approximation of Henyey-Greenstein scattering as a combination of an isotropic model and our strongly forward scattering model.

\section{IMPOSING THE BOUNDARY CONDITION}

In the preceding section, we introduced an approximation $G_{\mathrm{f}}$ for the propagator of an infinite volume forward scattering medium. Within computer graphics, we are mostly interested in using participating media as materials of (finite) objects, i.e. bounded volumes. In Sec. 3.2 we, therefore defined the propagator $G$ to have the absorbing boundary conditions of Eq. (3). In this section, we define a new 'bounded' propagator $G_{\mathrm{f}}^{\mathrm{b}}$ based on the infinite-volume result $G_{f}$, which (approximately) satisfies these boundary conditions.

\subsection{Dipole Models}

Given the linearity of the RTE, one can try to solve the boundary condition problem through the method of images. Within the diffusion approximation, simple dipole methods that introduce a single virtual source in order to approximately enforce a planar boundary are well known [Bell and Glasstone 1970; Lamarsh 1975]. These diffusion dipole solutions have been studied extensively in computer graphics [d'Eon 2014; Habel et al. 2013; Jensen et al. 2001].

As a remark, note that due to the angularly course nature of the diffusion approximation, such dipole methods can only satisfy an exact boundary condition such as Eq. (3) in an approximate (but asymptotically consistent) manner. Indeed, Eq. (3) should hold for every inward pointing direction $\hat{\omega}$, but classical diffusion methods only work with fluence and its resulting vector irradiance as a coarse angular approximation [Jensen et al. 2001].

Contrary to dipole methods, we have a fully angularly resolved propagator at our disposal. In principle, this allows us more freedom to better approximate the exact boundary condition of Eq. (3). However, in this initial work, we limit ourself to a simple dipole configuration similar to the directional dipole model of Frisvad et al. [2014] as described in the following section. Because our model reduces to the result of diffusion theory in the asymptotic limit, using such a classical dipole setup ensures asymptotic consistency.

\subsection{Dipole Setup}

We locally approximate a medium with general curved boundaries by a simple half-space with planar boundary through the dipole setup that is illustrated in Figure 2. The subscript on directions denotes whether they are at the incoming or outgoing point and the superscript denotes an incident or exitant direction. We use the same tangent plane as Frisvad et al. [2014] with modified normal

$$
\hat{n}_{\mathrm{i}}^{*}= \begin{cases}\hat{n}_{\mathrm{i}} & \text { if } \vec{x}_{\mathrm{o}}=\vec{x}_{\mathrm{i}}, \\ \frac{\vec{R}}{|\vec{R}|} \times \frac{\hat{n}_{\mathrm{i}} \times \vec{R}}{\left|\hat{n}_{\mathrm{i}} \times \vec{R}\right|} & \text { otherwise, }\end{cases}
$$

where $\vec{R}=\vec{x}_{\mathrm{o}}-\vec{x}_{\mathrm{i}}$ and the virtual source is similarly defined through

$$
\vec{x}_{\mathrm{v}}=\vec{x}_{\mathrm{i}}+2 A d_{\mathrm{e}} \hat{n}_{\mathrm{i}}^{*} \quad \text { and } \quad \hat{\omega}_{\mathrm{v}}=\hat{\omega}_{\mathrm{i}}^{\mathrm{e}}-2\left(\hat{\omega}_{\mathrm{i}}^{\mathrm{e}} \cdot \hat{n}_{\mathrm{i}}^{*}\right) \hat{n}_{\mathrm{i}}^{*},
$$




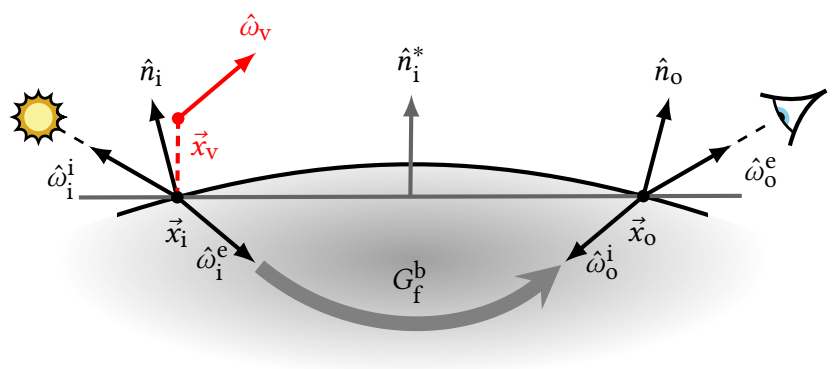

Fig. 2. Geometrical setup of the dipole model.

with extrapolation distance $d_{\mathrm{e}}$ given by $d_{\mathrm{e}}=2.131 \mathrm{D} / \sqrt{\alpha^{\prime}}$ for reduced scattering albedo $\alpha^{\prime}=\sigma_{\mathrm{s}}^{\prime} / \sigma_{\mathrm{t}}^{\prime}$ with reduced transmission coefficient $\sigma_{\mathrm{t}}^{\prime}=\sigma_{\mathrm{s}}^{\prime}+\sigma_{\mathrm{a}}$ and diffusion constant $D=1 /\left(3 \sigma_{\mathrm{t}}^{\prime}\right)$. The boundary condition of Eq. (3) corresponds to an index matched boundary in the classical diffusion dipole case (i.e. there is no internal Fresnel reflection at the boundary), which amounts to setting $A=1$. Lastly, the modified normal $\hat{n}_{\mathrm{i}}^{*}$ becomes ill-defined if $\hat{n}_{\mathrm{i}}$ is parallel to $\vec{R}$, in which case we simply set the propagator to zero.

The directional dipole model of Frisvad et al. [2014] additionally uses an empirical distance correction to implicitly offset the real source away from the boundary. Note that, as far as the geometry is concerned, there is no 'actual translation' of the source. The offset merely enters their BSSRDF as an effective regularization on the scalar distance to avoid singularities in the model. These singularities are an artefact of the diffusion assumption, which is only satisfied after distances much larger than the effective scattering length. By contrast, our propagator $G_{\mathrm{f}}$ only has a singularity 'by design' in that it reduces to the correct non-scattering delta distribution on short lengths (which can be accounted for with proper importance sampling), and is otherwise well-behaved. Hence, we need no such correction.

\subsection{The Final Boundary-Aware Propagator $G_{\mathrm{f}}^{\mathrm{b}}$}

By using the modified normal $\hat{n}_{\mathrm{i}}^{*}$ for the effective boundary plane, the incoming direction $\hat{\omega}_{\mathrm{i}}^{\mathrm{e}}$ can occasionally appear to point out of the medium, i.e. $\hat{n}_{\mathrm{i}}^{*} \cdot \hat{\omega}_{\mathrm{i}}^{\mathrm{e}}>0$. If the boundary were really planar, then the source would not illuminate the medium at all and there would be no transport towards the outgoing query point. We therefore choose a self-consistent resolution for this case by simply setting the propagator to zero.

Note that it is also possible for the outgoing direction to point into the effective half infinite medium. We found this to be less problematic compared to the incoming direction and do not handle this separately.

The final boundary-aware propagator then becomes

$$
G_{\mathrm{f}}^{\mathrm{b}}\left(s, \vec{x}_{\mathrm{o}}, \hat{\omega}_{\mathrm{o}}, \vec{x}_{\mathrm{i}}, \hat{\omega}_{\mathrm{i}}\right)= \begin{cases}0 & \text { if } \hat{\omega}_{\mathrm{i}} \cdot \hat{n}_{\mathrm{i}}^{*} \geq 0, \\ G_{\mathrm{f}}\left(s, \vec{x}_{\mathrm{o}}, \hat{\omega}_{\mathrm{o}}, \vec{x}_{\mathrm{i}}, \hat{\omega}_{\mathrm{i}}\right) & \text { otherwise, } \\ -G_{\mathrm{f}}\left(s, \vec{x}_{\mathrm{o}}, \hat{\omega}_{\mathrm{o}}, \vec{x}_{\mathrm{v}}, \hat{\omega}_{\mathrm{v}}\right) & \end{cases}
$$

with $\hat{n}_{\mathrm{i}}^{*}$ given by Eq. (12), $\vec{x}_{\mathrm{v}}$ and $\hat{\omega}_{\mathrm{v}}$ by Eq. (13), and $G_{\mathrm{f}}$ by Eq. (9).

\subsection{Reciprocity}

In concluding the discussion of our bounded propagator $G_{\mathrm{f}}^{\mathrm{b}}$, we mention its reciprocal properties, which are important when combining the model with bidirectional algorithms [Veach 1998].

From Eq. (9), it is apparent that the infinite volume propagator $G_{\mathrm{f}}$ is manifestly reciprocal, i.e. it is invariant under the exchange $\left(\vec{x}, \hat{\omega}, \vec{x}^{\prime}, \hat{\omega}^{\prime}\right) \leftrightarrow\left(\vec{x}^{\prime},-\hat{\omega}^{\prime}, \vec{x},-\hat{\omega}\right)$, which corresponds to changing the roles of emitter and receiver. However, the introduction of the virtual source in the bounded propagator $G_{\mathrm{f}}^{\mathrm{b}}$ breaks this symmetry, because $\vec{x}_{\mathrm{V}}$ depends on $\hat{n}_{\mathrm{i}}^{*}$, which itself does not depend on $\hat{n}_{\mathrm{i}}$ and $\hat{n}_{\mathrm{o}}$ in a symmetrical manner (in fact it is independent of $\hat{n}_{\mathrm{o}}$ ).

There are some configurations where the propagator $G_{\mathrm{f}}^{\mathrm{b}}$ does, however, show reciprocity. One particular case is when $\hat{n}_{\mathrm{i}}=\hat{n}_{\mathrm{o}}$, as occurs for planar boundaries. More generally, $G_{\mathrm{f}}^{\mathrm{b}}$ is reciprocal for every configuration where $\hat{n}_{\mathrm{i}}, \hat{n}_{\mathrm{o}}$ and $\vec{x}_{\mathrm{o}}-\vec{x}_{\mathrm{i}}$ are coplanar.

Reciprocity can be enforced for all configurations by defining a new reciprocal bounded propagator as the symmetrical combination

$$
\frac{1}{2}\left[G_{\mathrm{f}}^{\mathrm{b}}\left(s, \vec{x}_{\mathrm{o}}, \hat{\omega}_{\mathrm{o}}, \vec{x}_{\mathrm{i}}, \hat{\omega}_{\mathrm{i}}\right)+G_{\mathrm{f}}^{\mathrm{b}}\left(s, \vec{x}_{\mathrm{i}},-\hat{\omega}_{\mathrm{i}}, \vec{x}_{\mathrm{o}},-\hat{\omega}_{\mathrm{o}}\right)\right] .
$$

In practise, we found the difference between renders with the original propagator $G_{\mathrm{f}}^{\mathrm{b}}$ and this reciprocal combination to be negligible. Moreover, all results that we show in this paper are generated with unidirectional path tracing, and as such do not have a hard requirement of reciprocity. For these reasons, we use the simple $G_{\mathrm{f}}^{\mathrm{b}}$ propagator throughout the remainder of this work, instead of the combination in Eq. (15).

\section{IMPORTANCE SAMPLING THE FORWARD SCATTERING PROPAGATOR}

When rendering with our model, we have to evaluate the rendering equation (4) for the propagator $G_{\mathrm{f}}^{\mathrm{b}}$, i.e.

$$
\begin{array}{r}
L_{\mathrm{i}}\left(\vec{x}_{\mathrm{o}}, \hat{\omega}_{\mathrm{o}}^{\mathrm{i}}\right)=\int \mathrm{d} s \mathrm{~d} A\left(\vec{x}_{\mathrm{i}}\right) \mathrm{d}^{2} \hat{\omega}_{\mathrm{i}}^{\mathrm{e}} G_{\mathrm{f}}^{\mathrm{b}}\left(s, \vec{x}_{\mathrm{o}},-\hat{\omega}_{\mathrm{o}}^{\mathrm{i}}, \vec{x}_{\mathrm{i}}, \hat{\omega}_{\mathrm{i}}^{\mathrm{e}}\right)\left|\hat{\omega}_{\mathrm{i}}^{\mathrm{e}} \cdot \hat{n}\left(\vec{x}_{\mathrm{i}}\right)\right| \\
\times\left[L_{\mathrm{e}}\left(\vec{x}_{\mathrm{i}}, \hat{\omega}_{\mathrm{i}}^{\mathrm{e}}\right)+\int \mathrm{d}^{2} \hat{\omega}_{\mathrm{i}}^{\mathrm{i}} L_{\mathrm{i}}\left(\vec{x}_{\mathrm{i}}, \hat{\omega}_{\mathrm{i}}^{\mathrm{i}}\right) f_{\mathrm{s}}\left(\vec{x}_{\mathrm{i}}, \hat{\omega}_{\mathrm{i}}^{\mathrm{i}} \leftrightarrow \hat{\omega}_{\mathrm{i}}^{\mathrm{e}}\right)\left|\hat{\omega}_{\mathrm{i}}^{\mathrm{i}} \cdot \hat{n}\left(\vec{x}_{\mathrm{i}}\right)\right|\right],
\end{array}
$$

where we used variable names as in Figure 2 and assumed that we are in the steady state (i.e. dropping the $s$ dependence of $L_{\mathrm{i}}$ and $L_{\mathrm{e}}$ ).

We solve this rendering equation through standard Monte Carlo path tracing techniques by importance sampling a query point $\vec{x}_{\mathrm{i}}$ on the surface with directions $\hat{\omega}_{\mathrm{i}}^{\mathrm{e}}$ and $\hat{\omega}_{\mathrm{i}}^{\mathrm{i}}$ and a path length $s$. This section provides an overview of and some remarks on the strategy.

\subsection{Local versus Global Importance Sampling}

A standard path tracer for homogeneous media typically performs an exact local importance sampling at each scattering vertex through a sampling of the phase function and an exponential depth sampling based on transmission. However, such locally optimal sampling does not translate itself into a globally optimal sampling strategy, which should either take the full (unknown) volumetric radiance distribution into account [Křivánek and d'Eon 2014], or, equivalently, the radiance distribution on the boundary together with all scattering interactions at once. Because the number of scattering interactions in a 
path is unbounded, it effectively represents an infinite dimensional sampling problem, for which exact expressions quickly become intractable [Georgiev et al. 2013].

On the other hand, although the expression for $G_{f}$ in Eq. (9) might look somewhat daunting at first sight, the advantage over a traditional simulation of all scattering interactions is that its analytical form is completely known. Moreover, the rendering equation (16) only requires a seven dimensional sampling $\left(\vec{x}_{\mathrm{i}}\right.$ on a surface, $s, \hat{\omega}_{\mathrm{i}}^{\mathrm{e}}$ and $\hat{\omega}_{\mathrm{i}}^{\mathrm{i}}$ ) to capture the volumetric point-to-point transport, as opposed to the infinite dimensional standard path tracing problem. As such, one can in principle try to construct a perfect importance sampling of $G_{f}$, representing a global importance sampling of the volumetric transport.

In the current work, we have limited ourselves to analytical sampling schemes based on asymptotic approximations. As we will show, this approximate global importance sampling of our dipole model still provides a significantly faster converging estimator than the perfect local importance sampling of a traditional path tracer.

\subsection{Overview of our Importance Sampling Scheme}

The full details, formulas and derivations for the importance sampling scheme are provided in the supplementary material due to space limitations. This section gives a brief overview of the different sampling steps.

7.2.1 Sampling $\vec{x}_{\mathrm{i}}$. The importance sampling starts by selecting a point $\vec{x}_{\mathrm{i}}$ on the surface of the object. We use a one sample Multiple Importance Sampling (MIS) [Veach 1998] weighted combination of two methods. The first method samples an offset in a plane through the point $\vec{x}_{\mathrm{O}}$ and projects this point onto the surface. The second method samples a direction for $\vec{R}$ based on a marginalization of the short length weight of Eq. (10) and then shoots a ray along this direction to capture the initial ballistic peak.

These steps use computationally efficient, local decisions and ray traced projection to arrive on the surface. Because we only use local information (we do not 'know' the full geometry), this is the step over which we have the least control and hence we perform it first, while the propagator marginalized over the other sampling dimensions is still maximally broad and smooth ${ }^{2}$.

7.2.2 Indirect Sampling of $s, \hat{\omega}_{\mathrm{i}}^{\mathrm{e}}$ and $\hat{\omega}_{\mathrm{i}}^{\mathrm{i}}$. Once the point $\vec{x}_{\mathrm{i}}$ is known, the path length $s$ is sampled based on an MIS combination of analytical limit results for the directionally marginalized $G_{\mathrm{f}}$ in large and small $p s$.

With $\vec{x}_{\mathrm{i}}$ and $s$ known, $\hat{\omega}_{\mathrm{i}}^{\mathrm{e}}$ is sampled based on the exponential weight of $G_{\mathrm{f}}$ in Eq. (9). The boundary BSDF then uses $\hat{\omega}_{\mathrm{i}}^{\mathrm{e}}$ to sample an incident direction $\hat{\omega}_{\mathrm{i}}^{\mathrm{i}}$ (which can itself potentially point back into the medium when there is internal reflection).

7.2.3 Direct Sampling of $\hat{\omega}_{\mathrm{i}}^{\mathrm{i}}, \hat{\omega}_{\mathrm{i}}^{\mathrm{e}}$ and $s$. The previous, indirect method of sampling $s, \hat{\omega}_{\mathrm{i}}^{\mathrm{e}}$ and $\hat{\omega}_{\mathrm{i}}^{\mathrm{i}}$ is MIS weighted with a second approach that performs next event estimation by directly sampling a direction $\hat{\omega}_{\mathrm{i}}^{\mathrm{i}}$ towards a light source. This direction is then used

${ }^{2}$ A more optimal alternative could try to combine knowledge of $G_{\mathrm{f}}^{\mathrm{b}}$ with information about the actual, full geometry for the sampling. However, this would likely result in an increased computational cost compared to simply projecting rays, which is why we currently opt for the simple approach. by the adjoint BSDF to sample a direction $\hat{\omega}_{\mathrm{i}}^{\mathrm{e}}$ into the medium. A specialized path length sampler uses knowledge of $\hat{\omega}_{\mathrm{i}}^{\mathrm{e}}$ to generate a suitable length $s$.

\subsection{Robustness}

When our importance sampling scheme samples a direction that reflects back into the medium, we use a simple path splitting approach based on the path throughput so far in order to keep the throughput near unity (see e.g. [Vorba and Křivánek 2016] for a more elaborate approach). Likewise, a standard Russian roulette technique is used to keep the path throughput from becoming too small.

Moreover, although the importance sampling scheme that we present here covers the target function quite well, there is always the possibility of rare outliers that can be generated through an occasional bad sampling decision. For practical rendering purposes, it is therefore advised to employ some form of statistically robust estimator. In our particular rendering workflow, multiple independent, intermediate (low sample count) results were already stored for subsequent merging. Instead of simply averaging all results, we use a sample trimmed mean over each pixel, discarding the $1 \% \sim 5 \%$ highest and lowest samples and averaging the leftover [Wilcox 2005]. This trades a slight bias ${ }^{3}$ for a large reduction in variance, thereby reducing the overall error of the estimator.

\section{THE EFFECTIVE BRDF}

For optically dense objects that are approximately planar on length scales of the reduced scattering length $1 / \sigma_{\mathrm{s}}^{\prime}$, and which are lit with low frequency illumination, the effect of subsurface scattering can be captured by an effective Bidirectional Reflectance Distribution Function (BRDF) $f_{\mathrm{r}}$ [Nicodemus et al. 1977]. Indeed, for those points $\vec{x}^{\prime}$ on the surface where $G$ takes non-negligibles values in Eq. (4), the cosine factor $\left|\hat{\omega}^{\prime} \cdot \hat{n}\left(\vec{x}^{\prime}\right)\right|$ and incoming radiance will be approximately constant under these conditions. The only $\vec{x}^{\prime}$ dependence then comes from the propagator $G$ itself, which can be integrated separately. The result is equivalent to evaluating the propagator for a planar light source.

\subsection{Propagator for a Planar Source}

Because $G_{f}$ of Eq. (9) is a simple Gaussian distribution on $\vec{r}$, it can be analytically integrated over a planar tangent source a distance $d$ away from the query point $\vec{x}$ as measured along its surface normal $\hat{n}$ to give the plane source propagator

$$
\begin{aligned}
G_{\mathrm{f}}^{\mathrm{p}}\left(s, d, \hat{\omega}, \hat{\omega}^{\prime}\right)= & \frac{\pi N}{p^{2} F} \exp \left\{-C-D \hat{\omega} \cdot \hat{\omega}^{\prime}-\operatorname{Epd}\left(\omega_{n}+\omega_{n}^{\prime}\right)-F(p d)^{2}\right. \\
& \left.+\frac{E^{2}}{4 F}\left[2+2 \hat{\omega} \cdot \hat{\omega}^{\prime}-\left(\omega_{n}+\omega_{n}^{\prime}\right)^{2}\right]-\sigma_{\mathrm{a}} s\right\},
\end{aligned}
$$

with $\omega_{n}=\hat{\omega} \cdot \hat{n}$ and $\omega_{n}^{\prime}=\hat{\omega}^{\prime} \cdot \hat{n}$.

\footnotetext{
${ }^{3}$ Due to the law of large numbers, the distribution of pixel values in each intermediate result approaches a Gaussian distribution for sufficiently large number of intermediate samples. For such a symmetrical distribution, the bias introduced by a sample trimmed mean reduces to zero. Hence, the robust estimator is still consistent.
} 
Analogously to Eq. (14), the equivalent boundary aware, planar propagator $G_{\mathrm{f}}^{\mathrm{pb}}$ then takes the form

$$
G_{\mathrm{f}}^{\mathrm{pb}}\left(s, \hat{\omega}_{\mathrm{o}}, \hat{\omega}_{\mathrm{i}}\right)= \begin{cases}0 & \text { if } \hat{\omega}_{\mathrm{i}} \cdot \hat{n} \geq 0, \\ G_{\mathrm{f}}^{\mathrm{p}}\left(s, 0, \hat{\omega}_{\mathrm{i}}, \hat{\omega}_{\mathrm{o}}\right) & \text { otherwise, } \\ -G_{\mathrm{f}}^{\mathrm{p}}\left(s, d_{\mathrm{e}}, \hat{\omega}_{\mathrm{i}}, \hat{\omega}_{\mathrm{v}}\right) & \end{cases}
$$

where $d_{\mathrm{e}}$ and $\hat{\omega}_{\mathrm{v}}$ are the same as in Eq. (13).

\subsection{The BRDF}

For an index matched medium, the effective forward scattering BRDF $f_{\mathrm{r}}$ is immediately given as $f_{\mathrm{r}}\left(\hat{\omega}_{\mathrm{i}}^{\mathrm{i}} \leftrightarrow \hat{\omega}_{\mathrm{o}}^{\mathrm{e}}\right)=\int G_{\mathrm{f}}^{\mathrm{pb}}\left(s, \hat{\omega}_{\mathrm{o}}^{\mathrm{e}},-\hat{\omega}_{\mathrm{i}}^{\mathrm{i}}\right) \mathrm{d} s$.

The effective BRDF for a medium with non-trivial surface interactions $f_{\mathrm{s}}$ can be found through the recursive evaluation of the rendering equation (16), where the integral of $G_{\mathrm{f}}^{\mathrm{b}}$ over $\vec{x}_{\mathrm{i}}$ has been replaced with the evaluation of $f_{\mathrm{r}}\left(-\hat{\omega}_{\mathrm{i}}^{\mathrm{e}} \leftrightarrow-\hat{\omega}_{\mathrm{o}}^{\mathrm{i}}\right)$ and $\vec{x}_{\mathrm{i}}$ has been set to $\vec{x}_{\mathrm{o}}$. In practice, this means that we keep sampling the index matched forward scattering BRDF $f_{\mathrm{r}}$ and the medium boundary $\operatorname{BSDF} f_{\mathrm{s}}$ repeatedly until $f_{\mathrm{s}}$ generates a query direction that points out of the medium.

\subsection{BRDF Importance Sampling}

The overall structure of the importance sampling scheme in Sec. 7.2 can still be maintained, with the simplification that $\vec{x}_{\mathrm{i}}$ is simply set to $\vec{x}_{\mathrm{o}}$ in the first step, and the weights for the subsequent steps are based on the planar propagator $G_{\mathrm{f}}^{\mathrm{pb}}$ instead of the point propagator $G_{\mathrm{f}}^{\mathrm{b}}$. The full details are provided in the supplementary material.

Compared to sampling the full subsurface model which uses rays to project a tentative incoming projection onto the surface, sampling our effective BRDF can be performed in constant time. Moreover, the analytical convolution of the original propagator over the planar source effectively smoothens the integrand in the new rendering equation. Together with the reduced dimensionality, this facilitates importance sampling and reduces the required number of samples for an equal quality evaluation, which makes the equivalent BRDF a prime candidate for a level of detail hierarchy.

\section{RESULTS AND DISCUSSION}

We have implemented our forward scattering dipole model in the context of a path tracer in the Mitsuba renderer [Wenzel 2010] and tested it on a variety of synthetic and natural scenes. To facilitate reproducibility of our model, we provide source code on the project web page of this paper.

All results of the Jensen et al. [2001] and d'Eon [2014] ${ }^{4}$ dipole models have the reduced isotropic single scattering and unscattered transmission added, as these paths are not captured by those models.

\subsection{Semi-Infinite Medium}

We validate our model by comparing its predictions to reference solutions for a collimated light beam impinging on a semi-infinite medium with planar, index-matched boundary. The exitant radiance distribution on the plane is visualised in Figure 3 for several

\footnotetext{
${ }^{4}$ Index mismatched results for the dual beam model use refracted directions and Fresnel transmission scaling, but the virtual source parameters given by d'Eon [2014] were only fitted for the index matched case. Nonetheless, its results are surprisingly good.
}

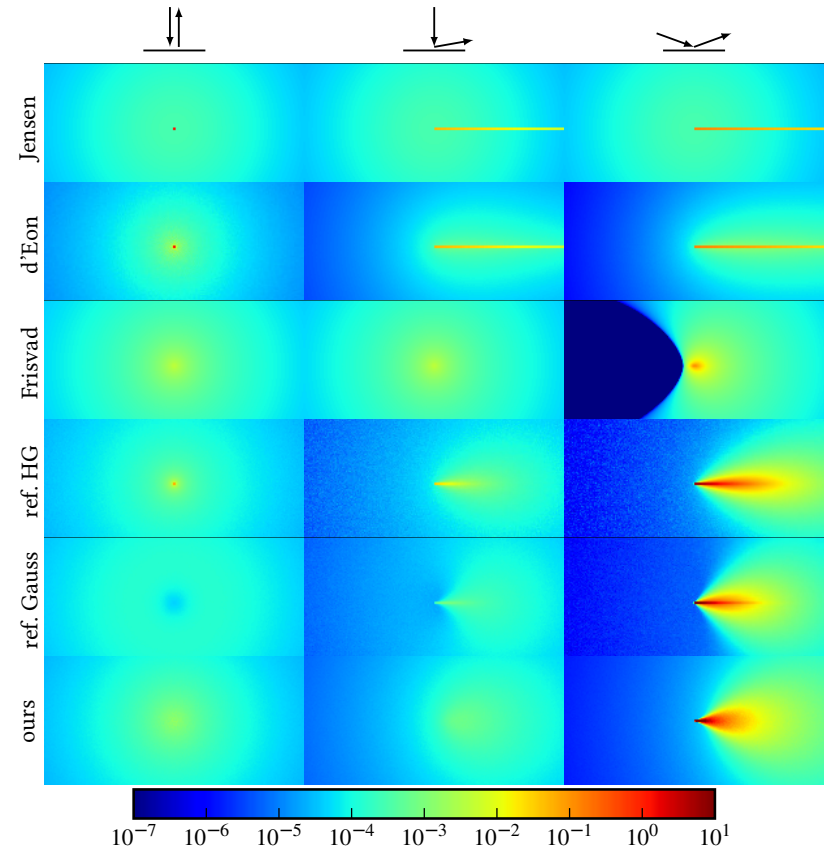

Fig. 3. Comparison of the exitant radiance pattern for a collimated beam incident on an index matched semi-infinite medium with planar boundary. The direction of incidence and exitance is visualized above the images and is either perpendicular to the boundary or at an $80^{\circ}$ angle to the normal. Dipole models are shown alongside light traced reference images with a HenyeyGreenstein (HG) and Gaussian phase function. The top two rows have isotropic single scattering added. The medium parameters are $\sigma_{\mathrm{s}}=1, \sigma_{\mathrm{a}}=$ $0.01, g=0.9$ and the visualized region covers the range $[-16,16] \times[-8.8]$.

incident and exitant directions. Note that the classical diffusion model of Jensen et al. [2001] is independent of incident or exitant direction, and the directional dipole model of Frisvad et al. [2014] only depends on the incoming direction. As a result, these models cannot correctly capture the strong, elongated forward scattering peak when the exitant direction starts to line up with the incident direction. The dual beam model of d'Eon [2014] is sensitive to both directions, but it inherently lacks the large forward scattering peak due to its assumption of isotropic scattering. Our model shows some backscattering in the first two columns akin to (a blurred version of) the reference solution for the Henyey-Greenstein (HG) [1941] phase function. In the forward scattering peak (third column), the behaviour tends more towards that of the Gaussian phase function.

In Figure 4 we provide 1D slices marginalized along the vertical axis of Figure 3, which confirms the correct handling of the forward scattering peak and the observation that our model is in a sense 'in between' the Gaussian and the HG behaviour. Note that there is no backscattering peak when the incoming and outgoing directions overlap (top middle and lower left image), which is consistent with the forward scattering assumption. Furthermore, the crucial forward scattering peak (lower right) is accurately reproduced, being two orders of magnitude larger than predicted by similarity theory.

Lastly, Figure 5 compares our effective BRDF against anisotropic references. In the forward scattering peak, our angular dependence 
resembles the reference Gaussian phase function result for $g \rightarrow 1$, as expected. Away from the peak, the correspondence with the HG reference is striking. The directional dipole model is also shown, but although it is explicitly dependent on the scattering anisotropy through the delta Eddington approximation, its directional behaviour is independent of the outgoing direction and thus it cannot reproduce the correct forward peak.

\subsection{Index Matched Media}

Figure 1 shows a simple scene with index matched media and a wide range of scattering parameters. The scene is backlit, emphasizing the strong directional properties of a forward scattering medium, as can be seen on the top of the cube. Our forward scattering dipole model matches the anisotropic ground truth images remarkably well. This is even the case for the optically thin medium on the right, where the majority of the error is manifested as a darkening on the hard edges of the cube due to the breakdown of the planar boundary approximation.

In contrast, the directional dipole model manages to approximately reproduce the overall brightness of the reference solutions, but it is unable to capture the directionality of the forward scattering peak on the top of the cube.

Note that, even for the optically dense scenes on the left where the conditions for applying the diffusion equation are justifiable, the application of similarity theory to transform to isotropic scattering clearly leads to incorrect results. On the other hand, our effective BRDF constitutes a remarkably accurate, yet computationally efficient approximation for these cases. This lends itself to a natural level of detail hierarchy with path tracing at the top, followed by our subsurface model, and finally our BRDF.

\subsection{Refractive Boundaries}

Diffusion based methods are expected to perform better for index mismatched boundaries, as the internally reflected light smooths out the angular flux near the surface. This aligns better with the modelling of transport by only a fluence and vector irradiance term.

Figure 6 shows the results for the well-known dragon model with a forward scattering material with index of refraction $n=1.6$. The difference between the diffusion methods and the ground truth is less pronounced than in the index matched scenes of Figure 1. This is partly because the assumptions underlying the diffusion approximation become less violated due to the internal reflection, and additionally because the refraction at the boundary forces a path to scatter over a larger angle in order to escape from the medium again, thus tempering the small-angle peak that we saw for the index-matched case. Nonetheless, our model still gives results that are closer to the path traced reference image, especially near the head and on the ridges of the tail.

In Figure 7, we show how our model performs for a spectral rendering of a realistic skin material. We use the dermis model of Donner and Jensen [2006] with $C_{\mathrm{h}}=0.01, C_{\mathrm{m}}=0.05$, and $B_{\mathrm{m}}=0.7$ to obtain spectral values for $\sigma_{\mathrm{s}}^{\prime}$ and $\sigma_{\mathrm{a}}$. The scattering anisotropy $g$ of human skin roughly varies between 0.7 to 0.8 for the dermis [VoDinh 2003]. We use the (spectrally constant) value of $g=0.75$ within a Gaussian phase function (a HG and isotropic reference are provided in the supplementary material). The index of refraction is set to $n=1.4$ and the interface is modelled with a Beckmann microfacet distribution of roughness $\sigma=0.35$, as per Donner and Jensen [2006]. Note that only our model and the reference solutions can make full use of this explicit interface description. The other dipole methods use the standard approach of scaling with the Fresnel transmission factors and changing the extrapolation length to simulate internal reflection. The results of our model show reasonable color accuracy compared to the reference image and the forward-scattered highlight on the side of the head is prominently present, as expected. However, the result is slightly too bright overall. This is similarly the case for the models of Jensen et al. and Frisvad et al. The dual beam model of d'Eon shows a reasonably accurate overall brightness, but has a distinct grey appearance compared to the ground truth.

In both scenes of Figure 6 and 7, the rendering time of our model is an order of magnitude longer (in an equal-quality comparison) than the simple classical dipole model of Jensen et al. combined with the exact planar importance sampling of Mertens et al. [2005]. This can be attributed to the inherently higher complexity of our model, an importance sampling that is only approximate, and the additional variance introduced from sampling the path length $s$. However, our technique provides more accurate results than the classical dipole model and is still an order of magnitude faster than the path tracing method (again, for equal quality). Also note that more advanced methods (e.g. bidirectional variants) do not outperform simple path tracing on these scenes, as the near-specular scattering interactions and high out-scattering loss in these volumes makes it practically impossible to connect paths. Moreover, for fixed $\sigma_{\mathrm{s}}^{\prime}$, the reference solution can be made arbitrarily hard by increasing $g$ further. Not only does the sampling problem itself become more singular, the sheer number of path vertices itself increases as well, rendering the computational problem doubly inefficient.

\section{LIMITATIONS AND FUTURE WORK}

In some scenes, such as in Figure 7, the overall brightness of our model was somewhat higher than that of the reference solution. It might be worthwhile to try and correct this effect by adjusting the virtual source strength and/or location to enforce a representative behaviour for a reference solution in a similar spirit as done by d'Eon [2014], ideally taking the anisotropy parameter $g$ into account. Indeed, it has been previously found that anisotropic scattering does have some influence on the ideal extrapolation length of a dipole model [Aronson 1995].

Contrary to previous dipole methods that are based on the angularly coarse diffusion approximation, we have a fully angularly resolved propagator at our disposal. In principle, this allows us more freedom to better approximate the exact boundary condition of Eq. (3) in a more manifestly self-consistent manner.

More hypothetically, our analytical approximation for the radiance inside a forward scattering medium, combined with the recent work on zero-variance, guided random walks [Krrivánek and d'Eon 2014; Meng et al. 2016] could be an interesting direction for future research. These zero variance methods are as of yet only derived from the assumption of isotropically scattering media, and a combination with our model could give rise to an efficient and unbiased rendering technique for the particularly difficult case of strongly forward scattering participating media. 

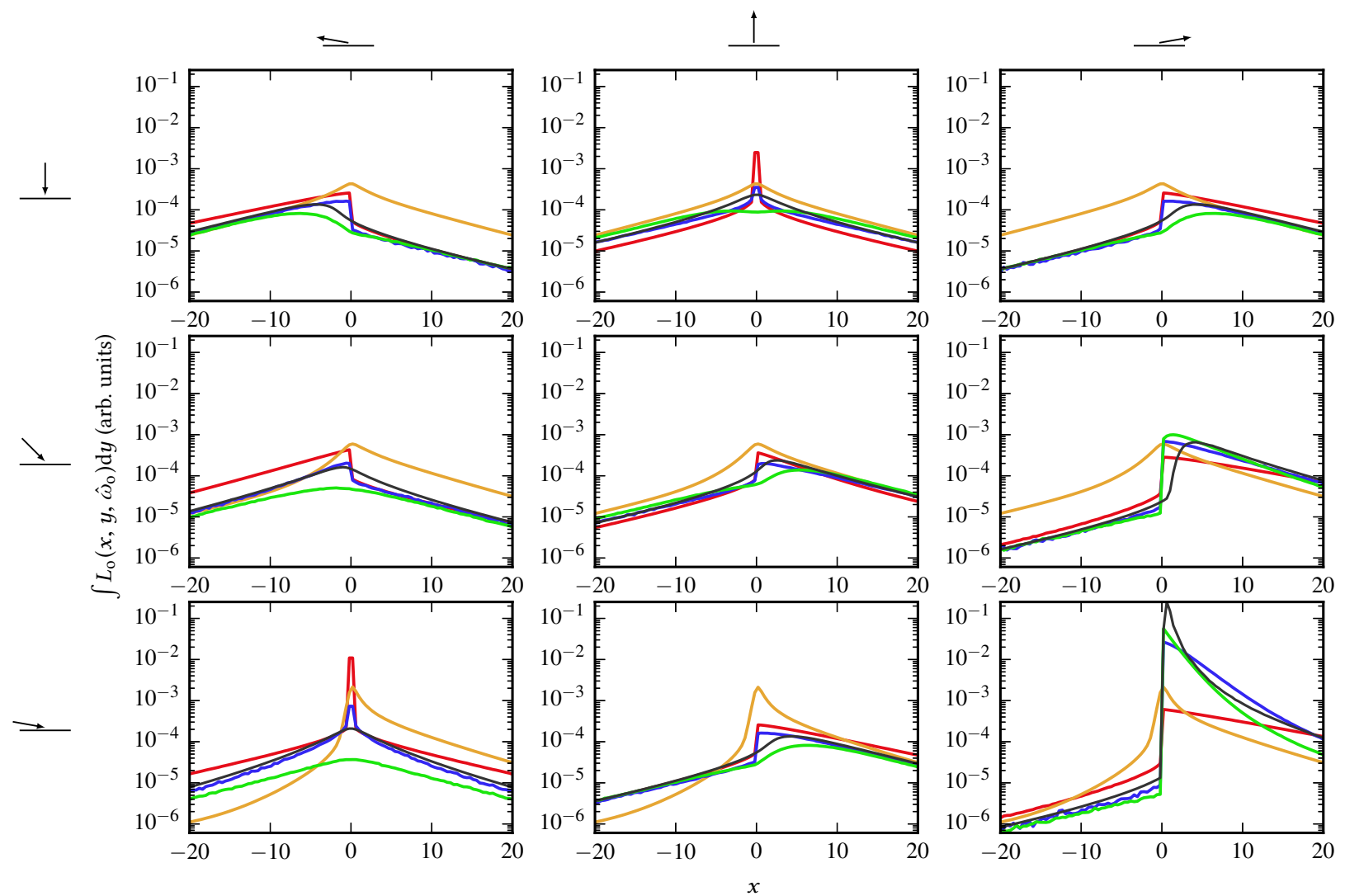

Fig. 4. Validation result showing one dimensional marginalized slices of the exitant radiance pattern for a semi infinite medium. The setup is identical to Figure 3, with the $x$ axis chosen along the horizontal direction and the marginalization performed along vertical slices of Figure 3. The predictions of our forward scattering dipole (black) are compared to reference results for a Henyey-Greenstein (blue), Gaussian (green) and equivalent isotropic phase function (red), as well as the directional dipole model of Frisvad et al. (orange). Rows correspond to incident angles of $0^{\circ}, 45^{\circ}$ and $80^{\circ}$ with respect to the normal, and columns correspond to an exitant angle of 0 or $80^{\circ}$, as indicated by the symbols. The results of our model generally lie in between the Gaussian and Henyey-Greenstein reference solutions. Our model correctly captures the important forward scattering peak at large incident and outgoing angles (bottom right), for which isotropic scattering through similarity theory (red) gives a result that is off by two orders of magnitude.

\section{CONCLUSION}

We have presented a novel subsurface scattering dipole model based on a first-principles derivation starting from a functional integral formulation of the radiative transfer equation under the assumption of strong forward scattering. The resulting propagator or Green's function takes the form of a time-resolved Gaussian weight on spatial and angular variables, which explicitly gets coupled to the boundary conditions of the medium as specified by the BSDF. Rendering with the model proceeds through the importance sampling of a propagator-based rendering equation.

We have tested our model for simple scenes with index matched media and scattering parameters spanning two orders of magnitude. Even in the optically dense case with low absorption, where diffusion theory is a good approximation, the use of the classical similarity theory relation which translates anisotropic scattering parameters $\sigma_{\mathrm{s}}$ to reduced isotropically scattering parameters $\sigma_{\mathrm{s}}^{\prime}=(1-g) \sigma_{\mathrm{s}}$ can lead to significant errors. Our explicitly forward scattering dipole model correctly handles this case and stays very close to the reference solution. Our model needs no explicit unscattered or single scattering contribution and stays well-behaved towards the optically thin limit of zero scattering without requiring ad-hoc regularizations.

In more complex scenes with index mismatched boundary conditions, the difference between our model and the diffusion dipole models becomes less extreme. Nonetheless, our model still shows superior color accuracy and correctly captures the distinct phenomenology of strong forward scattering.

Lastly, we have identified several avenues for future work and we hope that we can bring a renewed interest into the application of functional integral methods within computer graphics.

\section{ACKNOWLEDGMENTS}

The authors would like to thank the anonymous reviewers for their helpful comments and guidance. The head and dragon model are provided through the courtesy of Infinite Realities and Stanford University, respectively. 

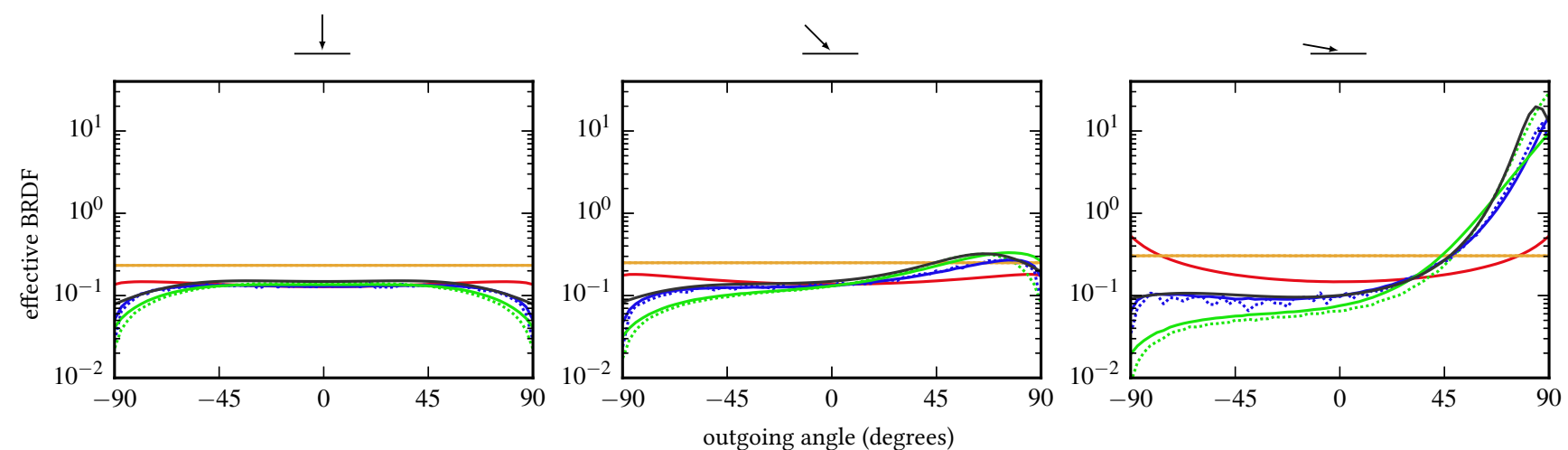

Fig. 5. Comparison of our effective BRDF (black) with reference solutions for HG (blue), Gaussian (green) and isotropic (red) phase functions, and the Frisvad et al. result (orange). The scattering parameters are $\sigma_{\mathrm{s}}^{\prime} / \sigma_{\mathrm{t}}^{\prime}=10 / 11$ as in the previous figures and the anisotropic references are given for $g=0.9$ (solid) and $g=0.99$ (dotted). The incoming direction makes an angle of $0^{\circ}, 45^{\circ}$ and $80^{\circ}$ with the normal as indicated above the plots, with the incoming and outgoing direction vectors in the same plane perpendicular to the surface. Away from the forward peak, the correspondence of our BRDF with the HG result is striking. In the forward scattering peak (rightmost image for large angles), our BRDF resembles the Gaussian phase function result for the highest anisotropy $(g=0.99)$, as expected from our Gaussian phase function assumption with $g \rightarrow 1$. Additionally, note that a rotationally invariant phase function implies mirror-symmetry about zero outgoing angle (red curves), but the result is not flat (diffuse), unlike what is often assumed in isotropic models (e.g. the orange lines).

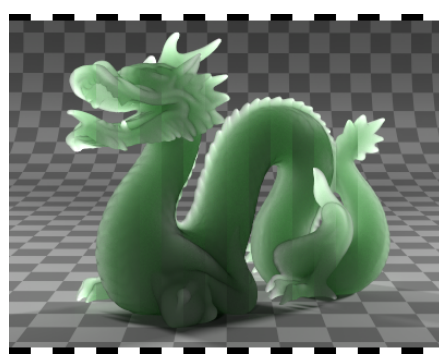

(a) Our model

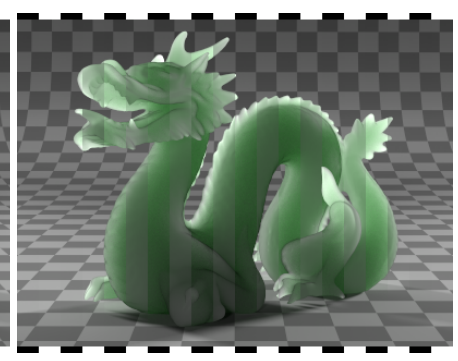

(b) Frisvad et al. [2014]

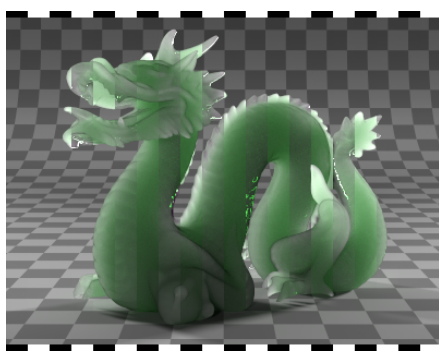

(c) d'Eon [2014]

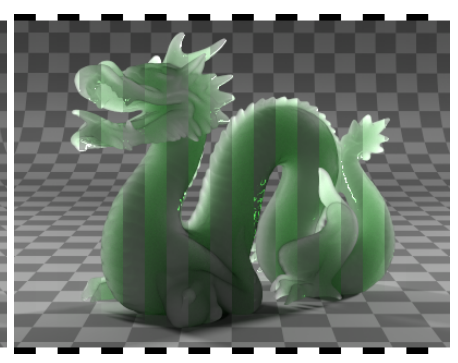

(d) Jensen et al. [2001]

Fig. 6. Comparison of dipole methods spliced together with the path traced reference solution for a unit length dragon with RGB scattering parameters $\sigma_{\mathrm{s}}=(400,300,400), \sigma_{\mathrm{a}}=(10,5,10), g=0.9$ and index of refraction $n=1$.6. The region that shows the reference solution is indicated by the black bars at the top and bottom of each image. External Fresnel reflections have been disabled for clarity and the reference solution uses a Gaussian phase function. Our model follows the reference solution particularly well and the difference is nearly indistinguishable on the front of the dragon. The other dipole models have particular trouble reproducing the deeply saturated green colour and maintaining the correct brightness in the thin regions such as the tail or the ridges on the back of the dragon.

\section{REFERENCES}

R. Aronson. 1995. Boundary conditions for diffusion of light. JOSA A 12, 11 (1995).

M. Ashikhmin, S. Premože, R. Ramamoorthi, and S. K. Nayar. 2004. Blurring of Light Due to Multiple Scattering by the Medium: A Path Integral Approach. Technical Report CUCS-017-04. Columbia University.

G. I. Bell and S. Glasstone. 1970. Nuclear Reactor Theory. Van Nostrand Reinhold Co.

E. Cerezo, F. Pérez, X. Pueyo, F. J. Seron, and F. X. Sillion. 2005. A Survey on Participating Media Rendering Techniques. Vis. Comput. 21, 5 (2005).

S. Chandrasekhar. 1943. Stochastic Problems in Physics and Astronomy. Reviews of modern physics 15, 1 (1943).

E. d'Eon. 2014. A Dual-beam 3D Searchlight BSSRDF. In ACM SIGGRAPH 2014 Talks (SIGGRAPH '14). ACM, Article 65.

E. d'Eon and G. Irving. 2011. A Quantized-diffusion Model for Rendering Translucent Materials. ACM Trans. Graph. 30, 4, Article 56 (2011).

C. Donner and H. W. Jensen. 2005. Light Diffusion in Multi-layered Translucent Materials. ACM Trans. Graph. 24, 3 (2005).

C. Donner and H. W. Jensen. 2006. A Spectral BSSRDF for Shading Human Skin. In Proceedings of the 17th Eurographics Conference on Rendering Techniques (EGSR '06). Eurographics Association.

C. Donner, J. Lawrence, R. Ramamoorthi, T. Hachisuka, H. W. Jensen, and S. Nayar. 2009. An Empirical BSSRDF Model. ACM Trans. Graph. 28, 3, Article 30 (2009).
O. Elek, T. Ritschel, and H.-P. Seidel. 2013. Real-Time Screen-Space Scattering in Homogeneous Environments. IEEE Computer Graphics and Applications 33, 3 (2013).

K. F. Freed. 1972. Functional Integrals and Polymer Statistics. Advances in Chemical Physics 22 (1972).

J. R. Frisvad, T. Hachisuka, and T. K. Kjeldsen. 2014. Directional Dipole Model for Subsurface Scattering. ACM Trans. Graph. 34, 1, Article 5 (2014).

I. Georgiev, J. Křivánek, T. Hachisuka, D. Nowrouzezahrai, and W. Jarosz. 2013. Joint Importance Sampling of Low-Order Volumetric Scattering. ACM Trans. Graph. (Proceedings of SIGGRAPH Asia) 32, 6 (2013).

I. Gkioulekas, S. Zhao, K. Bala, T. Zickler, and A. Levin. 2013. Inverse Volume Rendering with Material Dictionaries. ACM Trans. Graph. 32, 6, Article 162 (2013).

R. Habel, P. H. Christensen, and W. Jarosz. 2013. Photon Beam Diffusion: A Hybrid Monte Carlo Method for Subsurface Scattering. In Proceedings of the Eurographics Symposium on Rendering (EGSR '13). Eurographics Association.

L. G. Henyey and J. Leonard Greenstein. 1941. Diffuse radiation in the Galaxy. The Astrophysical fournal 93 (1941).

W. Jakob and S. Marschner. 2012. Manifold Exploration: A Markov Chain Monte Carlo Technique for Rendering Scenes with Difficult Specular Transport. ACM Trans. Graph. 31, 4, Article 58 (2012).

W. A. Jakob. 2013. Light Transport On Path-Space Manifolds. Ph.D. Dissertation. 


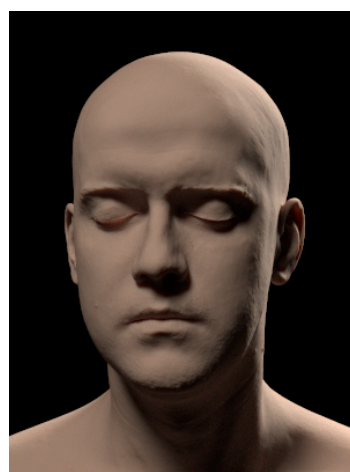

(a) Our model

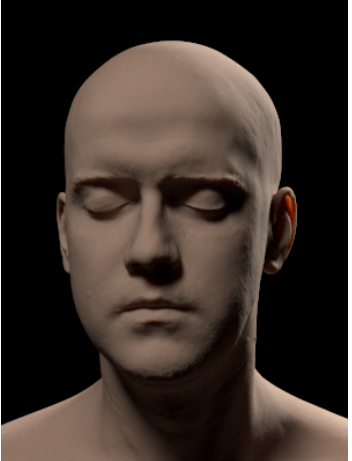

(b) Reference

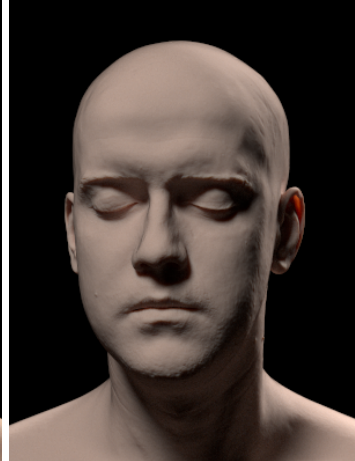

(c) Frisvad et al. [2014]

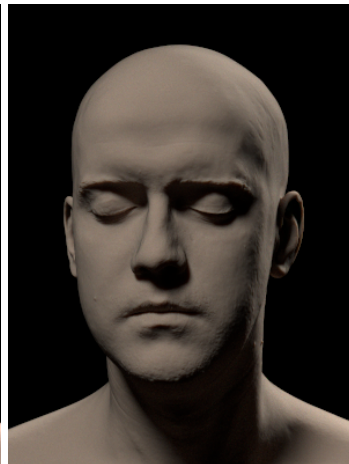

(d) d'Eon [2014]

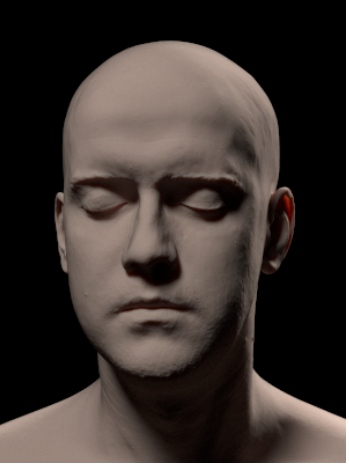

(e) Jensen et al. [2001]

Fig. 7. Comparison of dipole methods for a realistic spectral skin material with a Gaussian phase function of moderately high anisotropy $g=0.75$ (see the main text for the full parameters; external Fresnel reflections have again been disabled for clarity; additional results for HG and isotropic phase functions, as well as our BRDF, are provided in the supplementary material). The model is lit with a classical key and back light setup. Pay particular attention to the highlight from the back light on the side of the head and its relative intensity compared to the frontlit face. This highlight is the result of shallow angle scattering and thus especially sensitive to a forwardly peaked phase function. Our model accurately captures the colour of the path traced reference image, but our result is slightly too bright overall (similar to the related directional dipole model (c), but to a lesser degree). The diffusion based dipole models (d,e) tend towards an overly greyish, unsaturated tint and underestimate the relative intensity of the backlit highlight due to the assumption of isotropic scattering.

H. W. Jensen, S. R. Marschner, M. Levoy, and P. Hanrahan. 2001. A Practical Model for Subsurface Light Transport. In Proceedings of the 28th Annual Conference on Computer Graphics and Interactive Techniques (SIGGRAPH '01). ACM.

M. Kardar. 2007. Statistical Physics of Particles. Cambridge University Press.

P. Kilgo and J. Tessendorf. 2015. Accelerated Path Generation and Visualization for Numerical Integration of Feynman Path Integrals for Radiative Transfer. In foint International Conference on Mathematics and Computation, Supercomputing in Nuclear Applications and the Monte Carlo Method.

J. Křivánek and E. d'Eon. 2014. A Zero-variance-based Sampling Scheme for Monte Carlo Subsurface Scattering. In ACM SIGGRAPH 2014 Talks (SIGGRAPH '14). ACM, Article 66.

J. Křivánek, I. Georgiev, T. Hachisuka, P. Vévoda, M. Šik, D. Nowrouzezahrai, and W. Jarosz. 2014. Unifying Points, Beams, and Paths in Volumetric Light Transport Simulation. ACM Trans. Graph. (Proceedings of SIGGRAPH) 33, 4 (2014).

J. R. Lamarsh. 1975. Introduction to Nuclear Engineering. Addison-Wesley Pub. Co.

H. Li, F. Pellacini, and K. E. Torrance. 2005. A Hybrid Monte Carlo Method for Accurate and Efficient Subsurface Scattering. In Proceedings of the Sixteenth Eurographics Conference on Rendering Techniques (EGSR '05). Eurographics Association.

A. Liemert and A. Kienle. 2013. Exact and efficient solution of the radiative transport equation for the semi-infinite medium. Scientific Reports 3, 2018 (2013).

M. Machida. 2016. How to Construct Three-Dimensional Transport Theory Using Rotated Reference Frames. Fournal of Computational and Theoretical Transport 45, 7 (2016).

V. A Markel. 2004. Modified spherical harmonics method for solving the radiative transport equation. Waves in Random Media 14, 1 (2004).

F. Martelli, A. Sassaroli, A. Pifferi, A. Torricelli, L. Spinelli, and G. Zaccanti. 2007 Heuristic Green's function of the time dependent radiative transfer equation for a semi-infinite medium. Optics Express 15, 26 (2007).

J. Meng, J. Hanika, and C. Dachsbacher. 2016. Improving the Dwivedi Sampling Scheme. Computer Graphics Forum 35, 4 (2016).

T. Mertens, J. Kautz, P. Bekaert, F. Van Reeth, and H.-P. Seidel. 2005. Efficient rendering of local subsurface scattering. In Computer Graphics Forum, Vol. 24. Wiley Online Library.

M. F. Modest. 2013. Radiative Heat Transfer. Academic press.

S. G. Narasimhan, M. Gupta, C. Donner, R. Ramamoorthi, S. K. Nayar, and H. W. Jensen. 2006. Acquiring Scattering Properties of Participating Media by Dilution. ACM Trans. Graph. 25, 3 (2006).

F. E. Nicodemus, J. C. Richmond, J. J. Hsia, I. W. Ginsberg, and T. Limperis. 1977 Geometrical Considerations and Nomenclature for Reflectance. U.S. Government Printing Office.

J. C. J. Paasschens. 1997. Solution of the time-dependent Boltzmann equation. Physical Review E 56, 1 (1997).

M. S. Patterson, B. Chance, and B. C. Wilson. 1989. Time resolved reflectance and transmittance for the noninvasive measurement of tissue optical properties. Applied Optics 28, 12 (1989).
S. Premože, M. Ashikhmin, and P. Shirley. 2003. Path Integration for Light Transport in Volumes. In Proceedings of the 14th Eurographics Workshop on Rendering (EGRW '03). Eurographics Association.

S. Premože, M. Ashikhmin, J. Tessendorf, R. Ramamoorthi, and S. Nayar. 2004. Practical Rendering of Multiple Scattering Effects in Participating Media. In Proceedings of the Fifteenth Eurographics Conference on Rendering Techniques (EGSR'04). Eurographics Association.

A. J. Spakowitz and Z.-G. Wang. 2005. End-to-end distance vector distribution with fixed end orientations for the wormlike chain model. Physical Review E 72 (2005). Issue 4.

J. Tessendorf. 1987. Radiative transfer as a sum over paths. Physical Review A 35 (1987) Issue 2.

J. Tessendorf. 1989. Time-dependent radiative transfer and pulse evolution. JOSA A 6, 2 (1989)

J. Tessendorf. 1990. Radiative transfer on curved surfaces. F. Math. Phys. 31, 4 (1990).

J. Tessendorf. 1991. Underwater solar light field: analytical model from a WKB evaluation, In Underwater Imaging, Photography, and Visibility. Proc. SPIE 1537 (1991).

J. Tessendorf. 2003. Concepts for Volume Rendering. Technical Report. Rhythm \& Hues Studios.

J. Tessendorf. 2009. Numerical integration of the Feynman Path Integral for radiative transport (International Conference on Advances in Mathematics, Computational Methods, and Reactor Physics). American Nuclear Society.

J. Tessendorf and D. Wasson. 1994. 3D Cloud Scene Simulator V2 Algorithm for Scattering. Technical Report. Clemson University.

E. Veach. 1998. Robust Monte Carlo Methods for Light Transport Simulation. Ph.D. Dissertation.

T. Vo-Dinh. 2003. Biomedical photonics handbook. CRC Press.

J. Vorba and J. Křivánek. 2016. Adjoint-driven Russian Roulette and Splitting in Light Transport Simulation. ACM Trans. Graph. 35, 4, Article 42 (2016).

P. Weber, J. Hanika, and C. Dachsbacher. 2017. Multiple Vertex Next Event Estimation for Lighting in dense, forward-scattering Media. In Proceedings of the EUROGRAPHICS 2017 Conference.

J. Wenzel. 2010. Mitsuba Physically Based Renderer. (2010). http://www.mitsubarenderer.org/.

R. R. Wilcox. 2005. Introduction to Robust Estimation and Hypothesis Testing (2nd ed ed.). Academic Press.

R. G. Winkler, P. Reineker, and L. Harnau. 1994. Models and equilibrium properties of stiff molecular chains. The fournal of Chemical Physics 101, 9 (1994).

D. R. Wyman, M. S. Patterson, and B. C. Wilson. 1989. Similarity Relations for Anisotropic Scattering in Monte Carlo Simulations of Deeply Penetrating Neutral Particles. F. Comput. Phys. 81, 1 (1989).

A. Zee. 2003. Quantum Field Theory in a Nutshell. Princeton University Press.

S. Zhao, R. Ramamoorthi, and K. Bala. 2014. High-order Similarity Relations in Radiative Transfer. ACM Trans. Graph. 33, 4, Article 104 (2014). 